\title{
Reabilitação oral com prótese bucomaxilofacial em paciente pediátrica submetida à excisão de lesão neoplásica benigna em maxila
}

\author{
Oral rehabilitation by bucomaxillofacial prosthesis in pediatric patients submitted to resection of benign \\ neoplasm injury in maxilla \\ Rehabilitación oral mediante prótesis bucomaxilofacial en pacientes pediátricos sometidos a resección de lesión \\ por neoplasia benigna en maxilar \\ Olívia Breda MOSS $^{1}$ \\ Brena Carolina de Lima PINHEIRO ${ }^{1}$ \\ Tássia Caroline da Costa MENDES ${ }^{2}$ \\ Francisco Pantoja BRAGA ${ }^{3}$ \\ Brigitte NICHTHAUSER ${ }^{3}$ \\ Cristiane Maria Brasil LEAL ${ }^{3}$ \\ Graduanda em Odontologia pela Universidade do Estado do Amazonas (UEA), 69065-001 Manaus-AM, Brasil \\ Cirurgiã-Dentista, 69065-001 Manaus-AM, Brasil \\ ${ }^{3}$ Departamento de Prótese Total e Bucomaxilofacial da Universidade do Estado do Amazonas (UEA), 69065-001 Manaus-AM, Brasil
}

\begin{abstract}
Resumo
Mutilações faciais decorrentes de cirurgia oncológica provocam deformações na físionomia, levando os pacientes à depressão e ao isolamento social. Na impossibilidade de reconstrução cirúrgica, a reabilitação protética com próteses bucomaxilofaciais é o meio preferível para restabelecer a aparência estética e corrigir dificuldades de alimentação, deglutição e fonação, proporcionando reintegração social e melhorando a qualidade de vida. Este artigo visa relatar um caso de reabilitação com prótese obturadora maxilar em paciente pediátrico após cirurgia oncológica para tratamento de osteoma em palato, que provocou comunicação bucosinusal e perda parcial da maxila. Paciente do gênero feminino, 11 anos, procurou atendimento odontológico após cirurgia de excisão de um osteoma que originou comunicação bucosinusal. Após anamnese, exames intra e extra-oral e análise dos modelos de estudo optou-se pela realização de uma prótese parcial removível provisória superior com obturador palatino e grampos auxiliares de fios ortodônticos, pois a paciente encontrava-se em fase de crescimento. Após moldagem das arcadas superior e inferior, confecção do plano de cera superior e ajuste deste plano seguindo o perfil facial e oclusal da paciente, os modelos foram montados em articulador semi-ajustável e realizada a seleção e montagem dos dentes artificiais. A prova estética e funcional dos dentes mostrou o restabelecimento estético e fonético da paciente. Foi realizada moldagem funcional com boca fechada seguida da acrilização, instalação e proservações da prótese. Conclui-se que a prótese bucomaxilofacial superior vedou a comunicação bucosinusal, restabeleceu a estética, fonética e função mastigatória, devolvendo autoestima e reintegração social a paciente.

Descritores: Prótese Maxilofacial; Obturadores Palatinos; Prótese Dentária; Reabilitação Bucal.
\end{abstract}

\section{Abstract}

Facial mutilations resulting from oncologic surgery cause deformities in the physiognomy, leading patients to depression and social isolation. In the impossibility of surgical reconstruction, prosthetic rehabilitation with bucomaxillofacial prostheses is the preferable therapy of restoring aesthetic appearance and correcting feeding, swallowing and phonation difficulties, providing social reintegration and improving the quality of life. This article aims to report a case of rehabilitation with maxillary obturator prosthesis in pediatric patients after oncologic surgery for the treatment of osteoma in the palate, which caused bucosinusal communication and partial loss of the maxilla. A female patient, 11 years old, sought dental care after excision of an osteoma that caused bucosinusal communication. After anamnesis, intra- and extra-oral examinations and analysis of the study models, a temporary superior removable partial denture with a palatal obturator and auxiliary orthodontic wire clips was chosen, since the patient was in a growth phase. After molding the upper and lower arches, making the upper wax plane and adjusting this plane following the facial and occlusal profile of the patient, the models were mounted in a semi-adjustable articulator, the selection and assembly of the artificial teeth were performed. The aesthetic and functional test of the teeth showed the aesthetic and phonetic restoration of the patient. Functional molding was performed with closed mouth followed by acrylization, prosthesis installation and preservation. It is concluded that the superior bucomaxillofacial prosthesis closed the bucosinal communication, reestablished aesthetics, phonetics and masticatory function, restoring self-esteem and social reintegration to the patient.

Descriptors: Maxillofacial Prothesis; Palatal Obturators; Dental Prosthesis; Mouth Rehabilitation.

\section{Resumen}

Mutilaciones faciales resultantes de cirugía oncológica causan a los pacientes deformidades fisonómicas, depresión y aislamiento social. En la imposibilidad de reconstrucción quirúrgica, es preferible la rehabilitación con prótesis bucomaxilofaciales para restaurar aspectos estéticos y corregir las dificultades de alimentación, deglución y fonación, proporcionando reintegración social y mejorando la calidad de vida. Este artículo pretende presentar un caso de rehabilitación con prótesis de obturador maxilar en pacientes pediátricos tras cirugía oncológica para tratamiento del osteoma en el paladar, que causó comunicación bucosinusal y pérdida parcial del maxilar. Una paciente de 11 años de edad, buscó atención dental después de la extirpación de un osteoma que causó la comunicación bucosinusal. Tras anamnesis, exámenes intra y extraorales y análisis de los modelos de estudio, se eligió una prótesis parcial removible superior temporal con obturador del paladar e clips auxiliares de ortodoncia alambre, ya que el paciente estaba en crecimiento. Moldeados los arcos superior e inferior, hecho el plano de cera superior y ajustarlo siguiendo el perfil facial y oclusal de la paciente, los modelos se montaron en un articulador semiajustable, y se realizó la selección y el ensamblaje de los dientes artificiales. La prueba estética y funcional de los dientes mostró restauración estética y fonética. El moldeo funcional se realizó con la boca cerrada seguida de acrilación, instalación de prótesis y preservación. Se concluye que la prótesis bucomaxilofacial superior cerró la comunicación bucosinusal, restableció la estética, la fonética, la función masticatoria, restauró la autoestima y la reintegración social de la paciente.

Descriptores: Prótesis Maxilofacial; Obturadores Palatinos; Prótesis Dental; Rehabilitación Bucal.

\section{INTRODUÇÃO}

Os osteomas são neoformações osteoblásticas benignas compostas de osso maduro, compacto ou medular. São essencialmente restritos ao esqueleto craniofacial, portanto, raramente encontram-se em outros ossos. Os locais mais acometidos são, seio frontal, sendo o mais frequente, seguido do seio etmoidal, seio maxilar, seio esfenoidal, maxila e mandíbula, já sendo descrito na literatura caso de osteoma em arco zigomático ${ }^{1,2}$. Por apresentarem crescimento lento e ausência de sintomatologia dolorosa, o diagnóstico precoce é dificultado, ocorrendo este ocasionalmente devido exames 
odontológicos radiográficos de rotina. $\mathrm{O}$ tratamento de escolha é a remoção cirúrgica, sendo muito discutido o tipo de abordagem e a técnica utilizada ${ }^{3,4}$.

Uma vez que, pacientes submetidos à remoção cirúrgica da lesão podem apresentar sequelas que trazem prejuízos funcionais e estéticos, a utilização de reabilitação com próteses bucomaxilofaciais (PBMF) tornam-se extremamente benéficas para devolução do bem-estar social e qualidade de vida a estes pacientes ${ }^{5}$.

Entende-se por PBMF como uma substituição aloplástica de tecidos que foram perdidos na região dos maxilares e da face, a fim de restaurar a anatomia o mais próximo possível do natural. Tem a capacidade de reestabelecer as funções perdidas, embora em alguns casos existam limitações. Sua utilização está presente em reabilitações de pacientes que sofreram intervenções cirúrgicas mutiladoras, acidentes com arma de fogo, traumas, malformações congênitas e ainda, destruição óssea advinda do uso de drogas como a cocaína ${ }^{6}$.

Quando a intervenção se dá em área de maxila e a maxilectomia é realizada, várias abordagens terapêuticas têm sido publicadas, como enxertos não vascularizados, transferência de tecido livre, retalhos locais e regionais e obturadores protéticos. A reconstrução do defeito por retalho cirúrgico permite uma correção definitiva da comunicação bucosinusal, entretanto requer uma equipe e conhecimento técnico extremamente especializado ${ }^{7}$.

Já a confecção de uma prótese obturadora diminui o tempo de procedimento e oferece uma reabilitação mediata e adequada. Outra vantagem é a possibilidade de visualização do local da cirurgia, que pode facilmente ser examinado após a remoção da prótese obturadora, e caso haja uma recidiva da lesão, esta pode ser detectada facilmente e em tempo hábil ${ }^{7,8}$.

Deste modo, torna-se importante o conhecimento acerca dessa prótese e sua indicação, uma vez que sua confecção devolve ao paciente forma, função, estética, autoestima e reintegração social. Sendo assim, este artigo tem o objetivo de relatar um caso clínico de reabilitação com prótese parcial removível provisória superior com obturador palatino, após cirurgia oncológica para excisão de osteoma em palato, que resultou em perda parcial da maxila e uma comunicação bucosinusal.

\section{CASO CLÍNICO}

Paciente do gênero feminino, melanoderma, 11 anos, procurou a Policlínica Odontológica da Universidade do Estado do Amazonas/UEA para reabilitação dentária após ser submetida a maxilectomia parcial para excisão de um osteoma. $\mathrm{Na}$ anamnese constatou-se que a paciente gozava de boa saúde e não apresentava nenhum comprometimento sistêmico que pudesse comprometer o tratamento. Ao exame clínico extraoral verificou-se alteração facial do lado esquerdo, ausência de dor a palpação e inexistência de tumefação (Figura 1).

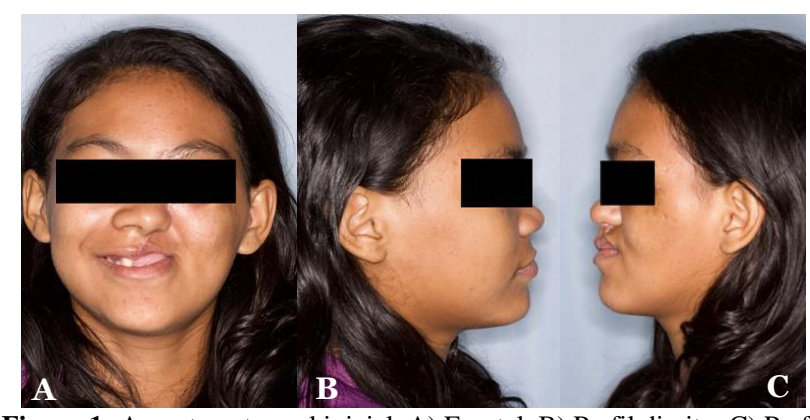

Figura 1: Aspecto extraoral inicial. A) Frontal. B) Perfil direito. C) Perfil esquerdo.

O exame clínico intra-oral revelou ausência dos elementos dentários 15,11,21-27, comunicação bucosinusal, ausência de cárie e de lesão periodontal (Figura 2). Este quadro clínico levou a paciente a relatar problemas funcionais na fonação, mastigação e deglutição além de insatisfação estética. Após análise dos exames, considerando que a paciente se recusava a qualquer intervenção cirúrgica reparadora e que se encontrava em fase de crescimento e de erupção de alguns elementos dentários, foi planejado como tratamento a confecção de uma prótese parcial removível provisória superior com obturador palatino e grampos auxiliares de fio ortodôntico para melhor estabilidade da prótese. $\mathrm{O}$ tratamento foi bem aceito pela paciente e sua mãe e o Termo de Consentimento Livre e Esclarecido (TCLE) foi assinado.

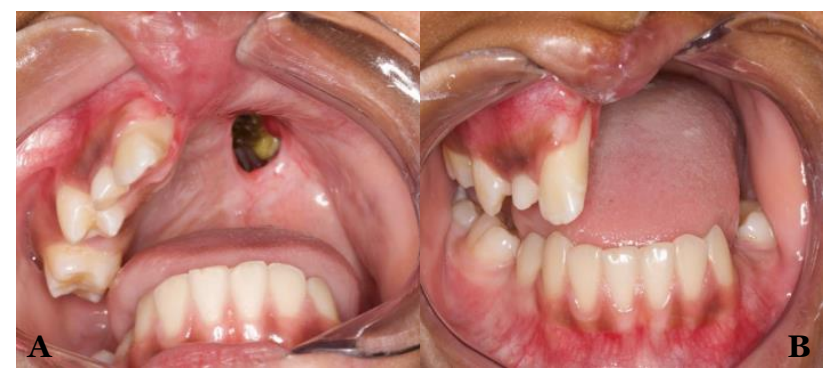

Figura 2: Aspecto inicial da cavidade bucal. A) Comunicação bucosinusal. B) Ausência de rebordo superior esquerdo após maxilectomia parcial.

$\mathrm{Na}$ arcada inferior os elementos dentários 34,35 e 45 estavam em fase de erupção. A moldagem anatômica das arcadas superior e inferior foi realizada com alginato, sendo a superior moldada com material em consistência mais espessa reproduzindo a extensão e profundidade da comunicação bucosinusal (Figura 3). No modelo superior foi confeccionado o plano de cera que posteriormente foi ajustado na boca da paciente seguindo seu perfil facial e oclusal (Figura 4). Após registro do arco facial, o modelo superior foi montado em articulador semiajustável e, em seguida, foram determinadas as relações intermaxilares para montagem do modelo inferior. Os dentes artificiais foram selecionados tomando como referência os dentes remanescentes da paciente, as linhas de 
referência demarcadas no plano de cera e características da paciente como sexo, formato do rosto e idade (Figura 5).

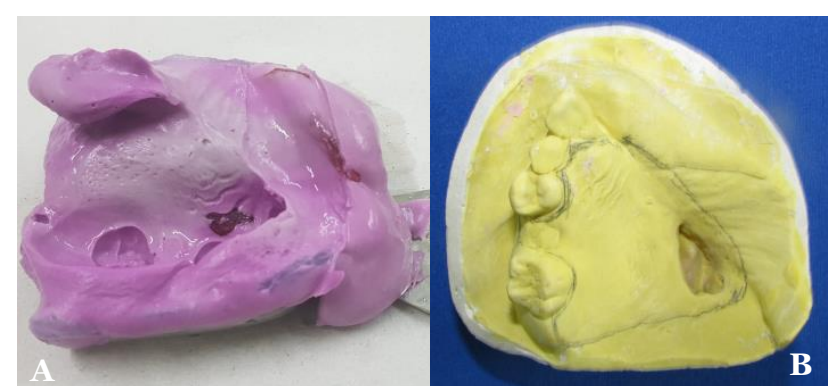

Figura 3: Molde e modelo da arcada superior. A) Molde superior. B) Modelo em gesso.

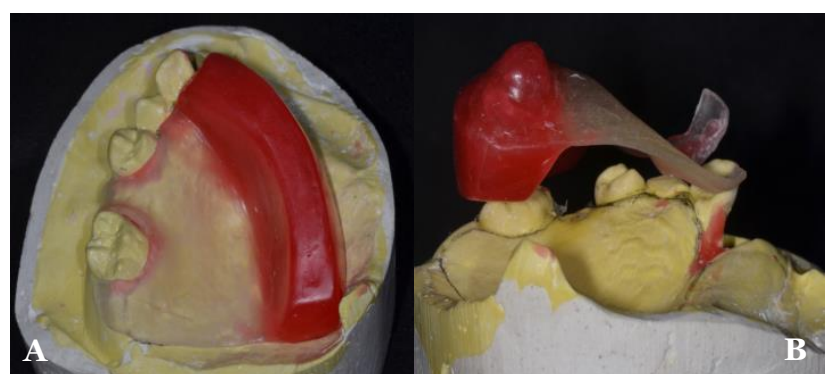

Figura 4: Modelo superior com plano de cera. A) Plano de cera lado esquerdo. B) Obturador palatino.

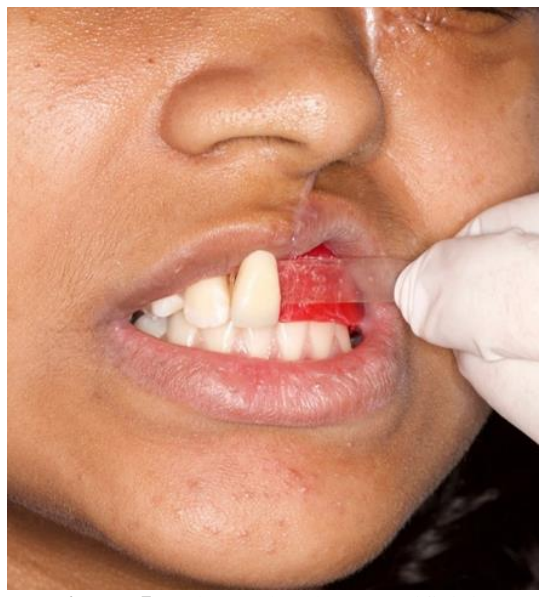

Figura 5: Seleção dos dentes artificiais.

Os dentes artificiais foram montados no plano de cera (Figura 6) e em seguida foi realizada a prova estética e funcional na cavidade bucal onde foram avaliados, suporte de lábio, altura incisal, corredor bucal, características dos dentes artificiais como cor, tamanho e formato, linha mediana, linha alta do sorriso, testes fonéticos, testes de retenção e estabilidade além de ajustes oclusais (Figura 7). Após ter sido constatado o restabelecimento estético, fonético e oclusal da paciente, foi realizada a moldagem funcional com boca fechada utilizando-se silicone de consistência leve. A prótese seguiu para as fases laboratoriais de acrilização, acabamento e polimento. Na instalação da prótese parcial removível provisória superior foram novamente realizados testes fonéticos, testes de retenção e estabilidade, análise dos contatos oclusais e verificados os princípios estéticos (Figuras 8 e 9).

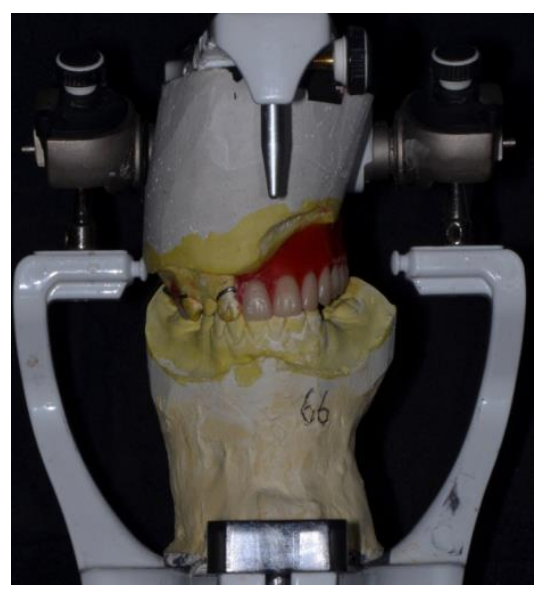

Figura 6: Dentes artificiais montados.
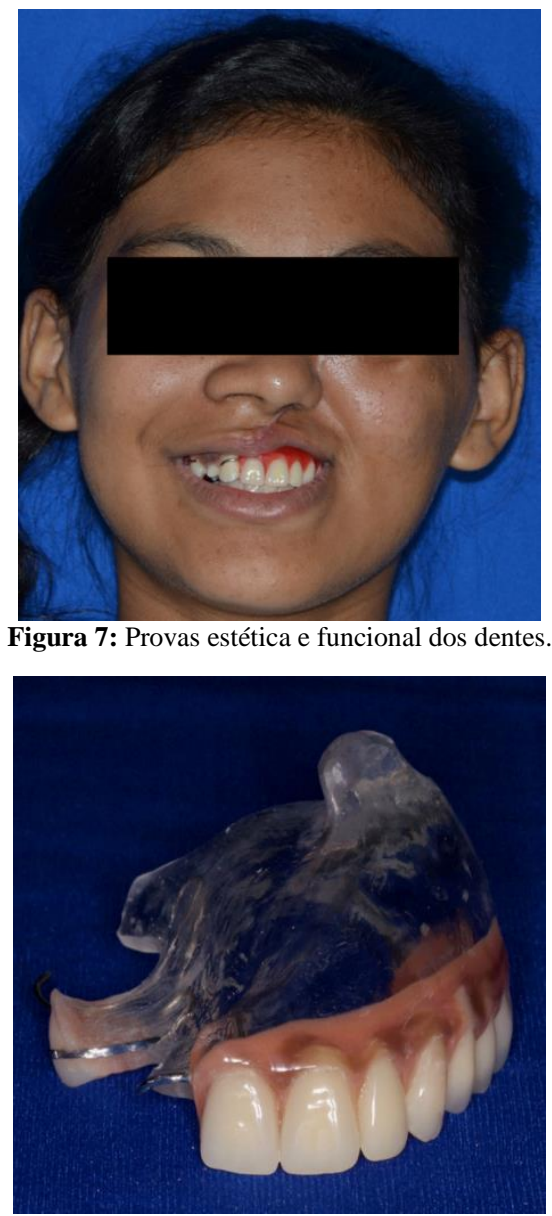

Figura 8: Prótese finalizada.

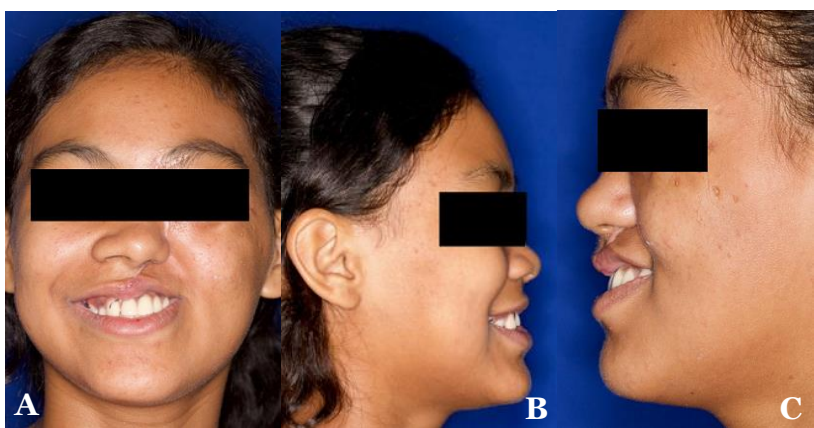

Figura 9: Aspecto clínico final. A) Frontal. B) Perfil direito. C) Perfil esquerdo.

A paciente recebeu por escrito informações sobre higienização e cuidados necessários para a manutenção da prótese. A primeira proservação foi 
realizada 24 horas após instalação, foram executados pequenos ajustes e a paciente relatou satisfação com a estética, com fala que se tornou inteligível e melhora significativa na deglutição e mastigação. Em seguida, foram realizadas proservações nos períodos de 48 horas e uma semana aonde foi constatada que a prótese ficou bem adaptada. A paciente recebeu acompanhamento no $15^{\circ}$ e $30^{\circ}$ dia após a instalação da prótese e em seguida foi orientada a retornar após três meses.

\section{DISCUSSÃO}

As mutilações bucomaxilofaciais possuem etiologia dividida em três grupos, congênitas causadas por más formações e distúrbios de desenvolvimentos, adquiridas ocasionadas por traumas ou acidentes, e de origem oncológica ${ }^{9}$. Estas mutilações causam deformidades bucomaxilofaciais, isto é, perda das formas originais na região de cabeça e pescoço, ocasionando comprometimento estético, funcional, psicológico e social ${ }^{10,11}$. Estes aspectos foram constatados e relatados pela paciente do caso clínico descrito ao procurar reabilitação bucal após ser submetida a cirurgia para excisão de osteoma.

$\mathrm{O}$ restabelecimento das estruturas faciais perdidas, pode ser realizado por meio de cirurgias plásticas reconstrutoras, utilizando-se enxertos teciduais ou retalhos, ou próteses bucomaxilofaciais $^{7,10,12}$. Quer sejam nasais, auriculares, oculares ou bucais, as próteses bucomaxilofaciais apresentam algumas vantagens sobre a cirurgia plástica, como reabilitação precoce, rapidez no restabelecimento da aparência, possibilidade de remoção e inspeção da área em caso de recidiva da neoplasia ${ }^{5,7}$

As maxilectomias parciais ou totais podem provocar comunicação bucosinusal gerando problemas fonéticos, alimentares e estéticos. A abordagem do caso clínico descrito seguiu a linha de estudo de Oliveira ${ }^{8}$, Rodrigues et al. ${ }^{9}$ e Chen et al. ${ }^{13}$ quando relatam que a prótese obturadora proporciona vedamento da cavidade bucal, promovendo o isolamento desta com a cavidade nasal, protegendo a região, evitando deposição alimentar, traumas, desconfortos, prevenindo a fala hipernasal, a regurgitação nasal de alimentos e líquidos e restabelecendo a sustentação do perfil facial. As próteses obturadoras podem ser imediatas, quando instalada logo após o procedimento cirúrgico ou mediatas, quando confeccionadas e instaladas após a cicatrização da cirurgia ${ }^{5}$.

$\mathrm{O}$ grau de recuperação funcional da prótese obturadora varia conforme as dimensões da comunicação bucosinusal, o número e a posição dos dentes, a altura do rebordo alveolar residual e extensão do assoalho palatino ${ }^{14}$. Além disto, podem ser utilizados meios de retenção como implantes osseointegrados e magnetos que auxiliam na retenção da prótese e assim, na recuperação funcional ${ }^{5,14}$. No caso clínico relatado, a paciente procurou reabilitação após cicatrização da cirurgia oncológica e por se encontrar em fase de crescimento foi realizada, como tratamento, uma prótese parcial removível provisória com obturador palatino e grampos com fio ortodôntico para auxiliar na estabilidade.

\section{CONSIDERAÇÕES FINAIS}

Mutilações bucomaxilofaciais apresentam etiologia congênita, traumática ou oncológica e provocam nos pacientes problemas estéticos, funcionais e psicológicos. Desta forma, o planejamento do tratamento reabilitador deve ser criterioso quanto à seleção do tipo de prótese bucomaxilofacial levando em consideração o estado sistêmico e emocional do paciente além de suas expectativas. No caso clínico relatado, a prótese parcial removível provisória superior com obturador palatino, vedou a comunicação bucosinusal, restabeleceu a estética, fonética e função mastigatória da paciente devolvendo-lhe autoestima e reintegrando-a ao convívio social. No entanto, faz-se necessário um acompanhamento contínuo com a paciente até a conclusão da fase de crescimento e execução do tratamento reabilitador com prótese parcial removível convencional com obturador palatino.

\section{REFERÊNCIAS}

1. Longo F, Califano L, De Maria G, Cicarelli R. Solitary osteoma of the mandibular ramus: report of a case. J Oral Maxillofac Surg. 2001; 59(6):698-700.

2. Akmansu H, Eryilmaz A, Dagli M, Korkmaz H. Endoscopic removal of paranasal sinus osteoma: a case report. J Oral Maxillofac Surg. 2002;60(2):230-32.

3. Silveira RL, Borges HO, Machado RA, Cancino CM, Oliveira MG. Análise imaginológica dos osteomas. Rev Bras Patol Oral. 2005;4(1):23-6.

4. Neville BW, Damm DD, Allen CM, Bouquot JE. Patologia oral e maxilofacial. Rio de Janeiro: Elsevier; 2016.

5. Kusterer LEFL, Paraguassú GM, Silva VSM, Sarmento VA. Reabilitação com obturador maxilar após cirurgia oncológica: relato de casos. Rev cir traumatol buco-maxilo-fac. 2012;12(4):9-16.

6. Contis SM, Pérez IM. Necesidad de prótesis bucomaxillofacial en pacientes atendidos em la consulta de somatoprótesis. Rev cuba med mil. 2006;35(3): ID:lil-459350.

7. Goiato MC, Pesqueira AA, Silva CR, Gennari H Filho, Santos DM. Patient satisfaction with maxillofacial prosthesis. Literature review. J Plast Reconstr Aesthet Surg. 2009;62(2):175-80.

8. Oliveira BS. Prótese bucomaxilofacial na reabilitação de pacientes com tumores de boca: análise de casos e impacto na qualidade de vida 
[monografia]. Florianópolis: Universidade Federal de Santa Catarina; 2016.

9. Rodrigues RG, Rodrigues DS, Olivieira DC. Reabilitação com prótese bucomaxilofacial: revisão de literatura. RSM. 2019;5:20-27.

10. Shibayama R, Tossi R, Campaner M, Queiroz ME, Dallazen E. Reabilitação Protética de Paciente Maxilectomizados-Relato de Caso. Rev Odontol Araçatuba. 2016;37(2):9-16.

11.Rabelo DP, Tanure RP, Grajeda FM, Grossmann SM. Reabilitação Protética Oral em Paciente Oncológico. Rev UninCor. 2018;16(1):1-8.

12. Alhaji MN, Ismail IA, Khalifa N. Maxillary obturator prosthesis for a hemimaxillectomy patient: A clinical case report. Saudi J Dent Res. 2016;7(2):153-59.

13. Chen C, Ren W, Gao L, Cheng Z, Zhang L, Li S et al. Function of obturator prosthesis after maxillectomy and prosthetic obturator rehabilitation. Braz J Otorhinolaryngol. 2016;82(2):177-83.

14. Miracca RAA, Andrade Sobrinho J, Tanaka EMG. Magnetos na retenção de prótese conjugada óculo palpebral e obturador palatino. PCL Rev Ibero Am Prótese Clínica \& Laboratorial. 2004; 6(32):365-75.

\section{CONFLITO DE INTERESSES}

Os autores declaram não haver conflitos de interesse.

\section{AUTOR PARA CORRESPONDENCIA}

\section{Cristiane Maria Brasil Leal}

cbleal@uea.edu.br

Submetido em 05/08/2019

Aceito em 28/11/2019 